\title{
Case report of ovarian torsion in the first trimester of pregnancy
}

\author{
Dr. Medic Donalda Surducki* \\ *ORCID ID:https://orcid.org/0000-0002-9657-3908 \\ ${ }^{*}$ Designation: Dr.medic \\ *Department: Gynecology \\ *Author University: Carol Davila \\ *Country:Germany \\ ${ }^{*}$ Tel No:004915776926012
}

Submitted: 27 April 2020

Approved: 2 May 2020

Published: 4 May 2020

How to cite this article: Surducki MD. Case report of ovarian torsion in the first trimester of pregnancy. G Med Sci. 2020; 1(1): 008-009.

https://www.doi.org/10.46766/thegms.gyn.20042301

Copyright: ๑ 2020 Donalda S. This is an open access article distributed under the Creative Commons Attribution License, which permits unrestricted use, distribution, and reproduction in any medium, provided the original work is properly cited.

\section{ABSTRACT}

Ovarian torsion refers to the complete or partial rotation of the ovary on its ligamentous supports, often resulting in impedance of its blood supply. It is one of the most common gynecologic surgical emergencies and may affect females of all ages. Torsion of the ovary in the pregnancy is very rare and it commonly occurs during the third trimester. It is frequently associated with ovary stimulation in In-vitro-fertilisation(IVF)or Ovary masses. Here we present the case of a 33 year-old GII PI with 11 weeks +2 days of pregnancy with right abdominal pain. Gynaecologic examination detected a circa $5 \mathrm{~cm}$ cyst and the torsion of the right ovary. Laparascopy and right salpingo-oophorectomy were performed.

\section{Introduction}

Ovarian torsion involves torsion of the ovarian tissue on its pedicle leading to reduced venous return, stromal edema, internal hemorrhage, and necrosis.(6) It is a rare condition in the pregnancy and it is tipically detected in the right side. The left side is thought to barely have space because of the sigmoid colon. Diagnosis is usually difficult, especially in the first trimester. The role of Magnetic Resonance Imaging (MRI) is debated.(2)

\section{Case report}

A 33 year-old GII PI presented to the emergency unit at 11weeks+2 days of pregnancy based on the last menstrual bleeding with a onset of severe right abdominal pain non-responding to analgesia. Pain was isolated in the right iliac fossa, non-radiating and not relieved by any position. Patient denied recent sexual intercourse, sudden movements. There was no history of IVF, previous torsions. In the gynecologic examination there was no discharge or vaginal bleeding. Potassium hydroxide $(\mathrm{KOH})$ negative. Transvaginalsonography and Transabdominal ultrasound (because of the pregnant uterus with TVS was hard to detect the adnexa) scan reported a circa $5 \mathrm{~cm}$ cyst and ovarian torsion on the right side. (fig2)There was evidence of free intraperitoneal fluid collection. Transabdominal ultrasound visualized a foetus in utero with normal development for the gestational age. (fig1) Paracetamol and Dipidolori.v provided no relief of pain. 


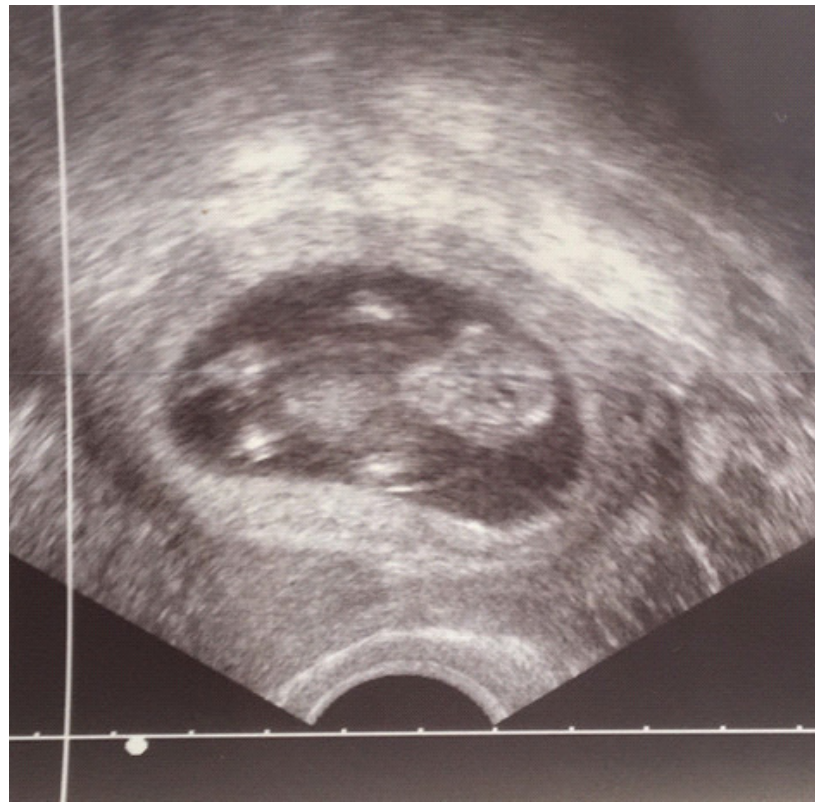

Fig 1

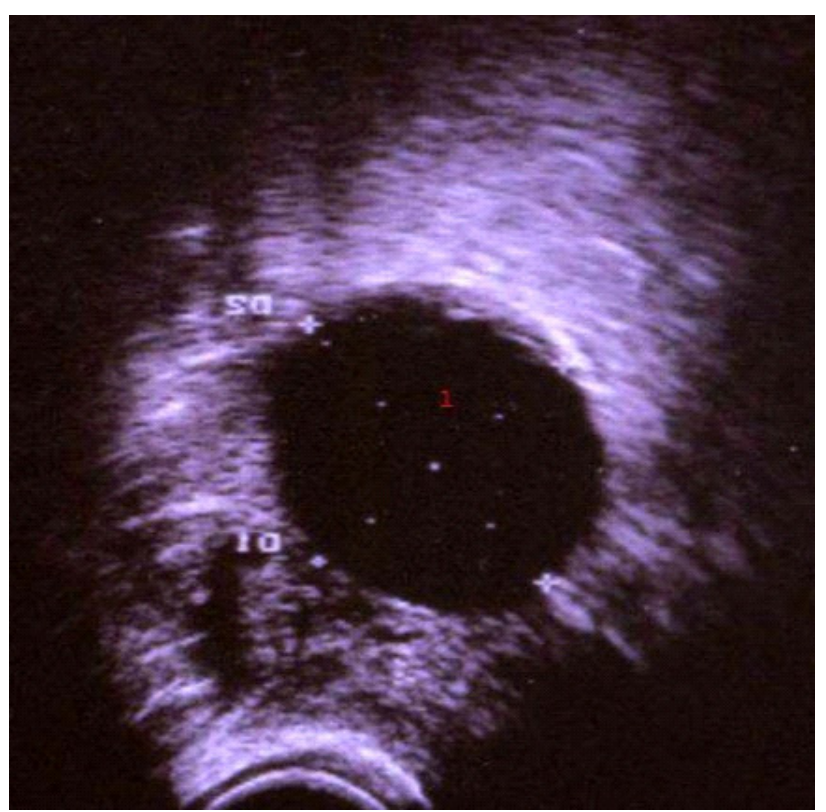

Fig 2

The patient was counseled concerning the risk of abortion and the possibility of adnexal torsion, and informed consent for laparoscopy and /or abdominal exploration with the possible need for salpingo-oophorectomy was obtained.

A Laparoscopy under general anesthesia was performed, demonstrating an enlarged, haemorrhagic, gangrenous , almost necrotic right ovary, twisted twice at the infundibulo-pelvic ligament (Fig. 3). Free intraperitoneal fluid collection was found. Detorsion was carefully performed, but no improvement in colour or decrease in edema was noted after $20 \mathrm{~min}$. A right salpingo-oophorectomy was then performed. The postoperative course was uneventful, and an intact intrauterine pregnancy was confirmed the next postoperative day.

Postoperative was the patient stable and free of pain. Progesterone oral was administrated till the end of 12 weeks of gestation.

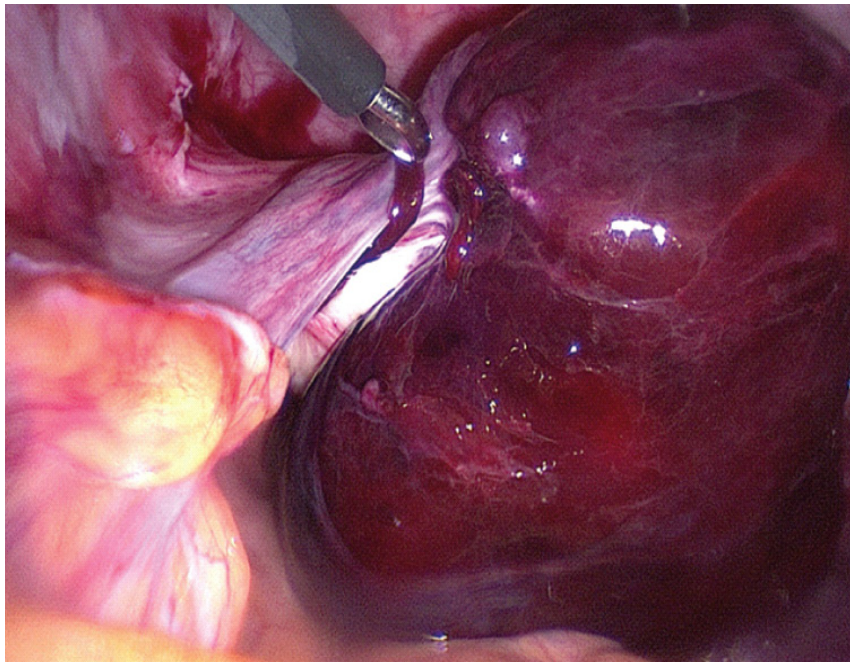

Fig 3

\section{Discussion}

Ovarian torsion during pregnancy is a rare condition, more common in the third trimester, and exceptional during the first trimester(1). The symptoms are nonspecific, and can be confused with other acute abdominal conditions such as appendicitis, ureteral or renal colic, cholecystitis and bowel obstruction.(4) (5) The presence on ultrasound of an ovarian mass should raise the suspicion for adnexal torsion. Management of ovarian torsion in pregnancy remains controversial.(2)(3) (4)(5) The laparoscopic approach combined with simple detorsion was tried but the salpingo-oophorectomy was necessary because of the failure of improvement in colour after detorsion. The preservation of the ovary was unfortunately not an option. We administrated Progesterone orally till the end of the 12 weeks. The patient had a normal pregnancy after that and delivered a healthy boy via C-section .

\section{Consent}

Written informed consent was obtained from the patient for publication of this case report and accompanying images.

\section{References}

1. Medscape (Torsion of normal adnexa definition)

2. C. Born, S. Wirth, A. Stäbler, M. Reiser.Diagnosis of adnexal torsion in the third trimester of pregnancy: a case report. Abdom Imaging, 29 (2004), pp. 123-127

3. A. Mancuso, G. Broccio, L.G. Angio, V. Pirri. Adnexal torsion in pregnancy 76 (1997), pp. 83-84

4. P.H. Huang, K.B. Tsai, E.M. Tsai, J.H. Su. Hemorrhagic corpus luteum cyst torsion in term pregnancy: a case report. Kaohsiung J Med Sci, 19 (2003), pp. 75-78

5. G. Oelsner, S.B. Cohen, D. Soriano, D. Admon, S. Mashiach, H. Carp. Minimal surgery for the twisted ischaemic adnexa can preserve ovarian function Human Reproduction, 18

6. Uptodate( definition of ovarian torsion) 